\title{
Perceived challenges by the Iranian baccalaureate surgical technology students in their clinical education: A qualitative study
}

\author{
H. Karimi Moonaghi ${ }^{1}$, R. Zardosht ${ }^{2 *}$, M. Etezad Razavi ${ }^{3}$ and S. Ahmady ${ }^{4}$ \\ ${ }^{1}$ Ph.D Nursing Education, Associate Professor, Evidence-Based Caring Research Center, Department of \\ Medical-Surgical Nursing, School of Nursing and Midwifery, Department of Medical Education, School of \\ Medicine, Mashhad University of Medical Sciences, Mashhad, Iran \\ ${ }^{2}$ Student Research Committee, School of Nursing and Midwifery, Mashhad University of Medical sciences, \\ Mashhad, Iran \\ ${ }^{3}$ Eye Research Center, Khatam-al-Anbia Hospital, Faculty of Medicine, Mashhad University of Medical \\ Sciences, Mashhad, Iran \\ ${ }^{4}$ Faculty of Medical Education, Shahid Beheshti University of Medical Sciences, Tehran, Iran
}

\section{ABSTRACT}

The operating room field in Iran is a branch of medical sciences during this period, students became become familiar with the new principles of the operation room and modern surgical techniques in specialized and super specialized surgeries and they train the care and assistance to the patient's administration before, during and after operation. The purpose of this qualitative study is to investigate how the students are taught, the effective factors and possible challenges related to educational issues in the operating room. This is a qualitative content analysis which was performed in 2014-2015. The participants consisted of 35 beneficiaries in the operating room field, namely, students of different academic terms, matrons, educators (instructors), surgeons and operating room personnel in different academic centers in Khorasan Razavi province who were rich in information and had a tendency to comment on their experience. In this study, sampling was first carried out in a targeted way and then was continued with theoretical sampling and sampling was continued until data saturation. All interviews were recorded and then handwritten and analyzed using continuous comparative method and qualitative content analysis. The profound and deep descriptions of the participants led to the emergence of 13 subcategories and 3 main categories of the "unconventional educational atmosphere", "the challenge of student admission to the surgical team," the "operating room culture" and, finally,

\section{ARTICLE INFORMATION:}

${ }^{*}$ Corresponding Author:

Received 21 June, 2017

Accepted after revision $27^{\text {th }}$ Sep, 2017

BBRC Print ISSN: 0974-6455

Online ISSN: 2321-4007 CODEN: USA BBRCBA

Thomson Reuters ISI ESC and Crossref Indexed Journal

NAAS Journal Score 2017: 4.31 Cosmos IF: 4.006

${ }^{\circ}$ A Society of Science and Nature Publication, 2017. All rights reserved.

Online Contents Available at: http//www.bbrc.in/

DOI: $10.21786 / \mathrm{bbrc} / 10.3 / 31$ 
one main theme of the "dynamic nature of the operating room".The special educational environment in the operating room environment, lack of acceptance of students in the surgical team as a training member and hierarchical culture of the operating room, has created problems for the training of these students. In fact, the clinical training of operating room students is based on teamwork and the members of the surgical team are, in some cases, instructors in the shade. Given the dynamic nature of the operating room, collaboration and participation between educational and clinical institutions can help improve the learning environment, acceptance students in the surgical team as a training member, and creating a dynamic, collaborative and flexible culture.

KEY WORDS: PERCEIVED CHALLENGES IRANIAN BACCALAUREATE SURGICAL TECHNOLOGY STUDENTS

\section{INTRODUCTION}

Bachelor of Science in surgical technology in Iran is a branch of medical science during which the students become familiar with new principles of surgical technologies in specialty and subspecialty surgeries and they learn how to care and help the patients before, during and after surgery (Operating Room and Anesthesia,2017). Training programs of operating rooms are offered in two ways worldwide: in the first case, nursing graduates (after achieving a bachelor's degree) are trained in a period of 1-2 years to enter the operating room. In the second case, surgical technicians, after general training of the operating room, are prepared to act as a mobile and scrubs and then, if desired, they are prepared as the first help of the surgeons in a period of $1 / 5$ to $2 / 5$ years. Standards of patient care in the community of operating room nurses in America also show that students of operating room technician can pursue their education in specialized courses in higher education. The people of each period have their own duties and the role of these specialized people is different from the operating room technician. Operating room is a complex system that coordinates the individual, technology, and patients in a physical environment to achieve favorable outcomes in patients (Islamic Republic of Iran Ministry of Health and Treatment and Medical Education Supreme Council for Planning of Medical Sciences, 2007). Operating room is considered as high-risk environment for patients, because in spite of the small number of cases, some potential problems of patients after the surgery are due to the mistakes of the operating room personnel that can lead to the death of the patients (Deyoung 2009).

In Iran, students of surgical technology are admitted directly based on the exam and with no relation to nursing. This field, in different colleges between the years 1987 to 2001 and it was launched at the undergraduate across the country, was launched in the associate degree level in 2009 (Operating Room and Anesthesia, 2017). Due to the fact that now in Iran there is no training courses in doctoral degree (Ph.D.) for surgical technology and its MA was launched only two years ago, MS nurses in internal injuries (with work experience in the operating room or an associate degree in surgical technology) take the responsibility of the practical and theoretical training of this group of students in educational centers. Given that the field is new and considering the volume increasing of training centers for students, it is not considered the required infrastructures such as specialist human resources in clinical training and educational facilities in proportion to the number of students. There is not enough number of teachers in the field, so in many centers teaching this group, traditionally, is given to the supervisors and operating room personnel. Subsequently, due to lack of necessary knowledge of educational affairs, students have faced with problems in their training (Bahrami et al 2014).

The purpose of the training is the optimal and effective care of patients before, during and after the surgery especially in the specialized surgeries, control and prevention of nosocomial infections as well as creating the perfect mental conditions for patients and proper maintenance of devices and medical equipment (Islamic Republic of Iran Ministry of Health and Treatment and Medical Education Supreme Council for Planning of Medical Sciences 2007). However, some items such as technical approach to the field, high expectation of the graduates in this field to help surgeons, and separation from nursing have caused the graduates to not have enough knowledge of nursing care of patients before and after surgery as well as communication with patients. So, they have just learned the technical skills during the surgery (Bailey 2010).

Foreign countries' researches have reported about training in the operating room which is connected with the collaboration (Silen-Lipponen et al., 2004) stress, communication failures (Laur et al., 2012), the effect of stress on other members and students (Kenton 2006) and to the development of the group of Skoczyl et al. (2005). The difference in the education of graduates of the surgical technology in Iran and other countries has caused the results of the studies in the field to be inapplicable in the country. Unfortunately, there is not extensive and efficient research in the surgical technology in the country. Conducted researches are quantitative and investigate certain aspects of education in surgical study. So, 
they are not able to analyze all aspects of education in the surgical technology (Bahrami et al., 2014; Khazaei et al., 2014, Tazakori et al., 2016).

However, qualitative approaches have holistic approach to the phenomenon and gain precious information about the training in the clinical environment. The aim of this qualitative content analysis is the evaluation of teaching the students, their required skills, effective factors and potential challenges associated with educational issues in the operating room. Planning to eliminate the defects of the field improves the knowledge and skills of students in this field, provides better care for patients, and ultimately improves the health system.

\section{MATERIAL AND METHODS}

The study is a qualitative content analysis study (part of a larger study). It was conducted in 2014-2016 to reveal the problems and challenges of education in the field of surgical technology in Iran. The environment includes all the operating rooms of educational hospitals of Khorasan Razavi (Mashhad, Nishabur, Torbat Heidarieh and Gonabad). The interview was done in places that are accessible to participants or places they desired. The participants were 35 of beneficiaries of education in surgical technology such as the students of different academic semesters, head nurses, teachers, surgeons and operating room personnel at various universities in Khorasan Razavi province. They have precious information and they were willing to recount their experiences. Sampling was done by using purposive sampling and continued by theoretical sampling. Some of the characteristics of the participants are as follows: willingness to participate in research and express their experiences, students who had spent at least one training courses, educators who had at least a year of training in the operating room, and operating room personnel who had at least one year of work experience in the room.

Data were collected by using semi-structured individual interviews. The interview was started by general questions regarding the interview directory and was guided by the responses of participants. After explaining the objectives of the plan to participants and obtaining informed consent, data collection was began. The main question were as follows: what makes you to call a day of the training as good and useful? Or feel the training is not of enough interest today? Mention a good experience and a bad experience from your training in the operating room? What are the facilitating and impeding factors in your clinical training?

A total of 37 interviews was carried out (35 initial interview and two follow-up interviews). The study continued from Tir 93 to Azar 95 (Iranian year). The researchers tried to consider the maximum variation in the sampling; (14 students, 6 Operation room personnel with associated degree or BS in surgical technology or BS in nursing, 4 operating room heads, 6 training teacher, one Dean of the Faculty, 3 surgical assistants and one surgical staff). Educational centers of Khorasan Razavi included Mashhad, Torbat Heydarieh, Gonabad and Nishabur. Data were analyzed according to conventional content analysis.

The data analysis was based on Graneheim and Lundman in seven steps (Graneheim and Lundman 2004). In the first step, all interviews were recorded and transcribed. Then, the decision made about the analysis unit and before starting the coding process, text of interviews was read several times as the unit of analysis. Meaning Units were also read and reviewed several times and unrelated statements were deleted. First, some units were defined and relevant codes of each Meaning unit were written and then the codes were classified inductively and based on common sense and meaning.

The process of data reduction was continued in all units of analysis and primary and secondary classes. In other words, subclasses with similar events are grouped together as classes and then primary classes formed and this trend continued up to the theme. It should be noted that during the analysis the necessary changes were done in the name of the classes which must show the content of the class. The analysis was continually conducted by adding up each interview and the classes were modified. To simplify the process of data analysis,

\begin{tabular}{|l|c|c|c|c|}
\hline $\begin{array}{l}\text { Work experience in the } \\
\text { operation room }\end{array}$ & Gender & Age & Number & Participants \\
\hline Semester 4 to 8 & 11 females/3 males & $22-20$ & 14 & Student \\
\hline 3-20 years & 5 females/1 male & $48-35$ & 6 & Training teacher \\
\hline 8-25 years & 5 females/1 male & $55-32$ & 6 & Operating room personnel \\
\hline- & Male & 52 & 1 & Dean of the Faculty \\
\hline $4-5$ years & $\begin{array}{c}\text { Chanbge this year to } \\
\text { English Calender Male }\end{array}$ & $48-32$ & 3 & Assistant \\
\hline 17 years & Male & 53 & 1 & Staff \\
\hline 15- 29 years & Male & $55-32$ & 4 & Operating room head \\
\hline
\end{tabular}


coding, cataloging and classification, comparing various data frequently and retrieving quotes, we used software MAXQDA 10 (Mayring 2000).

To increase the reliability and validity of the results, we used the methods used by Cuba and Lincoln that were quoted by Polit and Hungler. The two researchers consider the four criteria of confirm ability, credibility, dependability and transferability as necessary for the consistency and strength of qualitative data (Burn and Grove 2007). Therefore, researchers have helped to validate data with continuous involvement with data, data verification by the participants, allocation of sufficient time to the study and open communication and empathy with the participants. In order to determine the dependability, two separate research teams coded the Interviews that there was a high degree of agreement $(85 \%)$ between the comments. Also, great care was done in the collection, implementation and recording of data and allocating of enough time to collect the data. As for the transferability, manuscripts, interviews and units of analysis with extracted initial codes were provided to participants and the necessary corrections made and the proposed points were considered. In addition, three expert in the field of qualitative research monitored the study. Using a combination of methods (interviews and field notes) as well as sampling with maximum diversity (interviews with students of different academic semesters from different training centers, faculty-member educators, non-faculty member educators, and surgeons with different expertise) have increased the confirm ability and transferability of the data. In addition, describing the studied background, providing necessary explanations about the participants and using of their direct quotations was also done in this regard.

Ethical considerations in this study include: obtaining ethical permits from Committee for Medical Research
Ethics of Medical Sciences of Mashhad (ethical code 940548), explaining the purpose of the study and the use of tape recorder, explaining the way of collecting data for the participants and satisfying them to cooperate, obtaining consent in writing for conscious participation and paying attention to the basics including the research explanation, identifying the purpose of the study, stating the criteria for sample selection, the benefits of participation in the research, ensuring the anonymity, answering questions, the right to refuse to continue the research, providing adequate information and assuring the participants to put the interviews in a safe place.

\section{RESULTS AND DISCUSSION}

Data analysis of participants' statements and notes in the field lead to the emergence of 12 subcategories and 3 main categories and eventually one main theme.

\section{Main category: unconventional educational atmosphere}

This category includes the following subcategories: lack of facilities and trainers, lack of balance between trainings and goals, red lines, safety and sterility principals, the confrontation of the caring and technical roles of students and the simultaneous presence of other students.

Lack of facilities and teachers: learning environment has been considered as a key environment for education and training of students and for helping to link theory with the practice in the clinical setting. The data in this study showed that the participants are not satisfied with their learning environment. A training teacher with several years of experience in the operating room, says: "In the classroom sometimes it is required to explain or draw a part of something and there is no whiteboard and facilities there. There is no place for equipment and

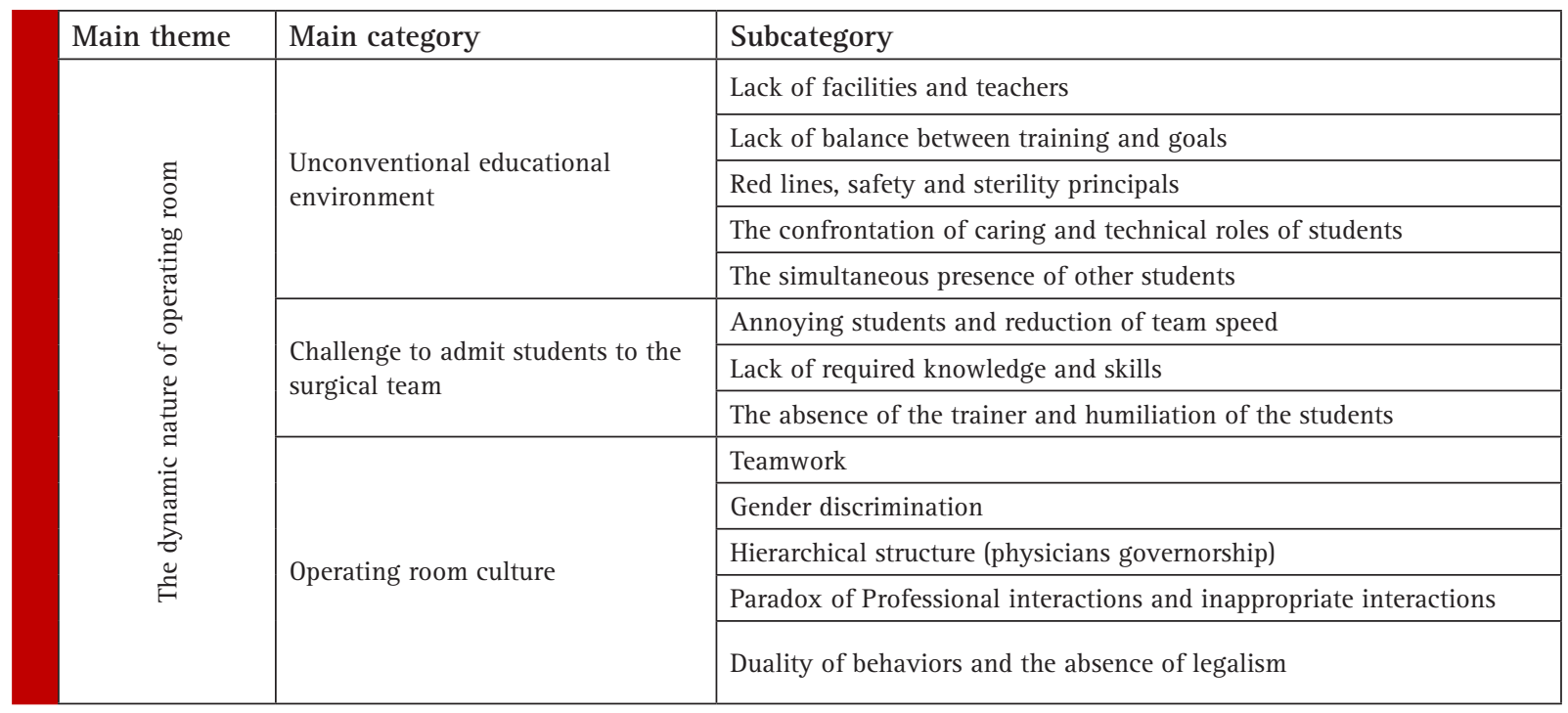


relaxation of students and it seems as though they do not have facilities for us". The conversion of associate degree to bachelor and the increase of the number of students regardless of the required infrastructure have created problems. The operating room head who has been in charge of training courses says: "The teacher cannot afford to train the students due to the increasing number of students as well as the presence of students from semester 4, 6, 8,". A student says:" teachers cannot teach us everything and do not have time to do so".In educational centers of cities other than Tehran, students actually cannot see thoracic and cardiac surgeries, advanced neurological and eye surgery. "Due to the low number of operating rooms and lack of variety in surgeries, the trainings in the cities are the same and students see the same surgeries in the semester 8 that had seen in semester 2"

Lack of trainers: it is hard to understand the routine and special procedures of hospitals for educators who temporarily go to the hospital with students. Absence of the trainer or the use of non-professional teachers or staff have affected the clinical training of surgical technology students. A student says: "When we do not have any trainers, the only thing is the attendance. Then, they told us to go to the rooms and it is not important to learn or not to learn”. In multiple studies, students' confusion and uncertainty in the absence of the trainer has been reported as factors causing problems.

"Of course, depending on the hospital, when we do not have trainer and the nurse is responsible for checking, there is no problem if we are late. I do not remember any washing. Because we do not have fixed trainer, they do not have us to do morning washing.

Mismatch between educational objectives and training objectives: Data analysis showed that the students complain about the aimless trainings and they consider the lack of transparency in goals and evaluating the training as a major problem. A senior student says: the lesson plan is originally for apprenticeship and they should it to students to understand what they do. In fact, I mean that we should be given a sheet based on which they evaluate us. I have not seen anything and most professors evaluate us based on scrub "Students considered as useless some training courses including recovery or management trainings. A student says: "We have weaknesses in file writing and recording of HIS and I personally cannot do this. At least they should include this in training courses. "A student told about the lack of training in Laparoscopy: "I went to the room for laparoscopic surgery and it was cholecystectomy. I did not know anything about laparoscopy even the theory. The personnel came and taught me quickly.

Red lines, safety and sterility principals: the operating room is a complex system in which an individual coordinates the technology and patients in a physical environment to achieve optimal outcomes in patients. Data analysis showed that the majority of operating room personnel are sensitive to safety and sterility principals of patients. A personnel says:

"If we told them to cover your hair, they would think it is because of Hijab but it is not and if the hair go to the sterile field, it can cause infection and problems for patients. Although we notify them, they hate. The health is important here and you can be as you will out of here. And you have to comply with the rules and standards here." On the other hand, surgeons also have their own reasons for patient safety: "The patient has trusted the surgeon about his life and the decision maker is the surgery team. Survival is not interchangeable with anything. It is not acceptable to put the life of the patient in danger."

Confrontation of caring roles and technical roles of students: in the operating room environment, compared to the other sectors, a specific aspect of care is mostly emphasized that is technical. One of the participants says:"If we look at it as a scientific discipline, we must go beyond the skills and consider it as theory-based thing. The problem here is that it seems most of the duties designated for the graduates of this field is practical, but this field is subdivision of the Nursing Science. The thing that now is happening is the technical look at the graduates of the field. So, they are expected to know the equipment and to help the surgeons in the surgery.

Simultaneous presence of students from other disciplines: the difference in the type of training and simultaneous activity of surgical technology and other students and operating team make the operating room training more different and difficult than other parts of the hospital. Faculty member trainer with 14 years of experience in training in the operating room says: "Simultaneous presence of students with different levels of training from freshmen to residents and even the professor himself in a place leads to different clinical experience in the place and the students cannot keep up with the situation and it is difficult to them.

\section{Second category: the challenge of admitting the students in the surgical team}

This category includes annoying students and the reduction of the team speed, lack of the required knowledge and skills of students, the absence of the trainer and the humiliation of students. Data analysis showed that the surgeon believes in the priority of patient safety and the life rather than the training and he/she seeks a surgery with minimal side effects for the patient.

Annoying students and the reduction of the team speed: an operating room personnel in this regard says:"The students are beginners and annoying. The 
personnel's works are rapid and easy, but it is not the same for students. They stand there without doing anything even when the assistant wants to move to the other place. Another trainer says:"Students in the operating room are uninvited guests and physicians and surgeons are sensitive to the students. Unfortunately, I'm concerned about the students and I permanently turn around in their room and it leads to sore feet because there is no place to sit.

Lack of required knowledge and skills of students: Interview with operating room assistants showed that admitting the students in the surgery team causes some problems."We had a student that knew no equipment. In the middle of the surgery we had to change him because it was a two hour surgery and we were just cutting the area without doing anything. He had lowered our speed and we had to change him.

\section{Absence of the trainer and the humiliation of students: a student says:}

"When we did not have the trainer, the personnel took the responsibility and the only important thing was the attendance. Then we were told to go to the rooms and it was up to us to learn or not to learn. "I thought that I am a useless creature that gets humiliated and the students were blamed for any mistakes."

Or another student talks about the impact of every single person on the admission of the student in the team: "The surgery began and we wanted to go to the room, but the surgeon did not allow. As I said it depends on the surgeon and other factors and all of these are effective in our training.

\section{Third category: The operating room culture}

This category includes teamwork, gender discrimination, hierarchical structure (surgeon as the governor), paradox of professional interactions and inappropriate interactions, behavior duality and lack of rule of law.

Teamwork: Since the operating room environment is of interactive and dynamic nature, to make the surgery team more cooperative and to eliminate the confusion of the students in the room, the clinical education program of the students should be transparent for the operating room personnel. Some operating room personnel do not consider the participation in the training as a part of their duties and even disappoint the students and the trainers with inappropriate behavior and create an unfavorable atmosphere. However, some staff play the role of the trainer for the students. "Most guys say that the educator plays an important role in the training. However, I say some personnel are the trainers of the students and they explain for you the techniques step by step in the operating room. Faculty member instructor with 14 years of work experience in training says: "See! It is a teamwork and each and every member of the operating room can have clinical effect. Perhaps the least role is for the head nurse and the personnel in the room have the greatest role in training students.

Gender discrimination: One of the known causes of stress in operating room environment for students is the others' behavior."A senior student says: unfortunately, some personnel or male surgeons work with female students. I like justice not discrimination. It is not fair to dismiss the female students earlier than males. If the female students cry, the personnel and the surgeon will change.

Hierarchical structure (doctor as the governor) researchers believe: a good clinical setting has non-hierarchical structure in which the works are done in teams or groups and there is good communication. A trainer says:"Look! When you have a pretentious assistant who does not know his work well and says I'm very good and am in a hurry, he does not let the students use the equipment correctly. He watches the student carefully. However, a patient assistant says take your time we are not in hurry and even tells anesthesiologist that we need more time. Unfortunately, we don't have many of this surgeons and you have to have a chance to work with them.

Paradox of professional interactions and inappropriate interactions: To make a team work so efficient, team members should be familiar with their capabilities. This leads to appropriate interactions between them and coordinates them. The trainer says:"Even students work more with good communicators. He might not be well in the knowledge, but he is good at communication. When he graduates, he is better than a good student with weak relationship and this is important. One of the operating room personnel in connection with the close interaction in the environment of operating rooms says: "some students abuse this open communication and some of them do not have the capacity of such communication.

Studies have shown that in a good clinical setting the interactions are not top-down and the work is done interactively. Data analysis and statements from students and educators in this study shows that surgeons have a top-down look to surgery team especially surgical technology students. This behavior is not in the framework of the operating room rules."Unfortunately, in Iran the higher the education, the higher the immorality and higher education leads to higher immorality.

Behavior duality and lack of rule of law: in the status quo operating room personnel do not consider the cooperation in training as their duty. And they do not have necessary cooperation with the trainer for providing the required facilities to students and transferring their experiences in the absence of the trainer."The student is of no position and is blamed for everything wrong even it is the fault of the personnel. Another trainer about the ignorance of some surgeons says:"According to the 
rules of the school we took a male student to the women operating room. The gynecologist of the room began to quarrel and said to force the student out. Another trainer in connection with the operation room atmosphere says:"There is no law here. If there is a law, they can be persecuted. However, doctors are the governors and we cannot blame them.

Results of the study show that the challenges of clinical education in the operating room in Iran, due to the complex and dynamic nature of operating room, are as follows: special educational environment, problems of admission of the students as a member of the surgical team in operating room, and different culture of the operating room. By comparing these, we can say that the certain circumstances, difference of the operating room, a shortage of faculty member in the department, and less control of the trainer in the clinical environment of the operating room have affected the training opportunities of students in this field.

Operating room (OR) clinical practice involves special contextual elements related to the preoperative nursing process, practical OR nursing skills and teamwork in different patient care situations. However, the OR is one of most inaccessible places in a hospital where the use of space is highly regulated. Areas of space are divided into unrestricted, semi restricted and restricted sections, and the physical activities performed within each of these designated areas are differentiated (Riley and Manias 2002). Working conditions for graduates of surgical technology is different than those of other nurses. He is forced to comply with a variety of procedures and high workload quickly and carefully. Due to the quick entry and exit of patients, his work is not expectable and in many cases he is faced with acute and emergency situations and concurrent interactions with members of the surgical team. It can harden the clinical education of the students (Farneya et al 2013).

Because of the special atmosphere of the operating room compared to other sectors, it seems that there is more emphasis on the students of the field in technical skills and specific approaches of the care. In Khazayi's study (2015) about the clinical education of surgical technology, the students gave the highest points to the learning opportunities and the lowest points to the performance of educators and educational facilities (Khazaei et al 2014). The high point to learning opportunities is due to the favorable reception in parts that were faced with shortage of human resources and the students played the role of assistance in these sectors. In the study of Ghorbanian et al.(2014), the most important weaknesses of clinical education of operating room are as follows lack of using teaching aids, inadequate number of patients for learning, lack of knowledge of students of clinical evaluation system, lack of adequate oversight on the clinical education and lack of decisionmaking power of the student in planning the care of patients (Ghorbanian et al., 2014).

These are consistent with the contents obtained in our research. In the study of Dehqani et al (2007), the absence of proper scientific grounds, lack of interest in the students, confusion and uncertainty of the students in the absence of the trainer were mentioned as factors causing problem for training process (Dehghani et al., 2005). Omidvar et al in Babol University of Medical Sciences also considered the problems of clinical education related to health centers' personnel and their knowledge (Omidvar et al. 2005).

In a similar study by Hadizadeh et al., it was found that the understanding and cooperation of the health centers staff is important in the educational process (Hadizadeh et al., 2005). Providing diverse learning opportunities, receiving proper feedback from trainers as well as clinical environment are the important and effective factors of clinical training in the other country's studies (Andrews et al., 2006 and 0'Driscoll et al., 2010). The results of this study showed that due to the special circumstances of the operating room, the admission of the students as a surgical team member has faced with difficulties.

Qualitative research of Lingard et al (2002) showed that the communication models in the operating room are complex and socially-motivated. The predominant themes include, time, security, sterilization, resources, roles and conditions. Often, every procedure had 1 to 4 stressful incidents and the stress transferred to other team members or the environment. In response to the tensions, the trainee of the operation was separated from the surgical team or began to mock. Both of these reactions are negative indicators for the team communications. The results are consistent with the study of Lingard et al (2002).

Tanner and Timmons (2000) emphasized that behavior in the OR is different from that in other hospital areas, and that the OR could be described as a 'backstage' area. In a backstage area interaction includes joking, talking about aspects of private life and behaving in a familiar manner. It also includes gossiping and disrespectful talking, even about patients during narcosis, which was also mentioned in this study. Frontstage behavior is the absence or opposite of this and occurs in the or when the patients are conscious before anesthesia or in the recovery unit (Tanner and Timmons 2000).Perhaps this antagonism between backstage and frontstage behaviors explains the students' excitement and frustration in the OR. Usually, before their OR placement period, students have spent time in frontstage areas, have dealt with staff more formally and have had closer caring relationships with patients. In addition, some nursing students have previous experience of patient care before enrolling in 
the programme (Manninen 1998(which probably makes their views about nursing more frontstage-oriented

In this study, participants have considered the admission to the surgical team as a gateway to opportunities for learning clinical skills and using experience of team members. However, the admission has its own problems. In the study of Tazkori et al (2015), issues related to clinical education in the operating room are as follows, the lack of proper planning for training period, lack of trust in teachers, lack of flexibility in the face of criticism and non-compliance with professional ethics and lack of taking time for clinical and educational consultation, lack of learning opportunities in hospitals, lack of familiarity with the described tasks of the students. In Liponen's study (2004) which was conducted as descriptive phenomenology associated with the clinical setting of operation room, three types of teamwork were reported from the point of view of students:

Three types of teamwork emerged based on the students' perceptions: functional manifestation of $\mathrm{OR}$ teamwork, gaining OR team membership and technical orientation of OR teamwork. The findings are discussed in relation to $\mathrm{OR}$ practice, education and research on ways to improve teamwork while maintaining a satisfactory OR learning context and stimulating interest in perioperative nursing). (Silen-Lipponen, et al 2004) The implications of this study is consistent with the findings of our study. In another study that was conducted in Bushehr University of Medical Sciences, the results showed that the students consider the lack of learning opportunities as one of the educational barriers (Abidi and Motamed 2011).

Although working in the operating room is a teamwork and it needs participatory culture, the results of our study showed the hierarchical culture in the clinical setting of the operating room and the surgeon is the commander and decision maker and sometimes he/ she loses the students' learning opportunities. Sadeghi et al (2011) believe that a weak and inflexible culture in the organizational environments causes employees not to tend to the innovation, change and creating new ideas and they fear from knowledge sharing. However, a dynamic, collaborative, and flexible culture reacts well to changes and leads to the progress (Sadeghi,,2011).

As studies show, participation and support from staff increase the learning opportunities for students (Heshmati et al2012). The results of another qualitative research (2004) showed that about 30 percent of the team transactions are faced with communication failure and cause problems. One-third of the cases associated with increased cognitive load (information processing in short-term and activated memory), interrupting the procedure, increased tensions and jeopardizing the safety of the patients (Zeighami et al 2004). However, Heshmatis study showed that nursing instructors use strategies like skills, realism, responsibility, determination, humility and using the experiences of nurses to attract the collaboration of clinical staff in providing clinical trainings (Heshmati 2012). However, other studies in our country showed that the nursing teachers have problems in playing their educational role (Heshmati et al 2010).

As stated by participants in this study, despite the difference and stressfulness of the clinical setting and its effect on clinical education of the students, participation and cooperation of every member of the surgical team can enhance the quality of the clinical skills of the students and this cannot be achieved unless in environments with supportive and participatory culture.This qualitative study was part of a larger study considering restriction of the studies with qualitative methodology; Such as: the subjective nature, field-based and their low level of generalizability. Despite these limitations, the results of this study provide deep understanding about the nature of clinical environment of the operating room and the problems related to clinical education of students of surgical technology.

\section{CONCLUSION}

According to the findings of this study, the unconventional educational environment in operating room, nonadmission of the student in the surgical team as an educational member, and hierarchical culture of operating room have caused problems for these students. Since the work in the operating room is collaborative and concurrent, control of this clinical learning environment is difficult. In fact, clinical education of the surgical technology students is team-based and all members of the surgical team are, somehow, trainers in the shadow. Due to the dynamic nature of the operating room, cooperation of educational and clinical institutions can improve educational environment, admission of the student as an educational member in the surgical team, and creating a dynamic, collaborative and flexible culture.

\section{ACKNOWLEDGEMENT}

This study is part of a larger study and extracted from a doctoral dissertation. Writers appreciate all the great professors who provide the research facilities as well as Deputy Minister for Research and Technology of Mashhad University of Medical Sciences for financial support.

\section{REFERENCES}

Abidi N, Motamed N. (2011). School of Allied Health and Nursing students' and teachers on the Bushehr University of Medical Sciences and Clinical Education. Strides Dev Med Educ; 8(1): 88-93. (Persian) 
Andrews GJ, Brodie DA, Andrews JP, Hillan E, Thomas BG, Wong J, Rixon L. (2006). Professional roles and communications in clinical placements: a qualitative study of nursing students' perceptions and some models for practice. International Journal Nurs Study; 43 (7):861-874.

Bahrami, T. Sadat, L. E. Mahmoudi. Golgini. E. (2014). Evaluation of the clinical training of students' views in the operating room and anesthesia Alborz. University of medical scienes and health .Journal of Alborz University of Medical Sciences. Volume 1, Number 3, summer 143-150, 139. [In Persian]

Bailey L. (2010). Strategies for decreasing patient anxiety in the perioperative setting. AORN J; 92(4): 445-57

Burns, N; Grove, SK.(2007). Understanding nursing research, building an evidence-based practice. 4th edition. St. Louis: Saunders Elsevier.

Dehghani H, Dehghani K, Fallahzadeh H. (2005). The educational problems of clinical field training based on nursing teachers and last year nursing student's viewpoints. Iranian Journal of Medical Education; 5(1):24-33. [Persian]

Deyoung S. (2009). Teaching strategies for nurse educators. 2th Ed. New Jersey: Prentice Hall. P.222-223.

Silen-Lipponen, M; Tossavainen, K; Turunen, H; Smith, A. (2004). Learning about teamwork in operating room clinical placement. British Journal of Nursing; 13.5 (24); 244-253.

Farneya, F; Abbaszadeh. A; Borhanif, F. (2013). inhibiting factors associated patient care in the operating room: a qualitative content analysis. Journal of Health Sciences Research; 2 (1): 90-7.

Ghorbanian N, Abdollahzadeh Mahlani F, Kazemi Haki B. (2014). Effective Factors on Clinical Education Quality; Anesthesiology and Operating Room Students View. Bimonthly Educe Strategy Med Sic; 6 (4):235-239. [In Persian]

Graneheim UH, Lundman B. (2004). Qualitative content analysis in nursing research: concepts, procedures and measures to achieve trustworthiness. Nurse Educ Today; 24(2): 105-12.

Hadizadeh F, Firoozi M, Shamaeyanrazavi N. (2005). Gonabad's nursing students' views about clinical Education assessment. Iranian Journal of Medical Education; 5(1):70-8. [Persian\}

Heshmati, Nabavi1, F. Vanaki, Z., Mohammadi, E. (2012). A Critical Review on Communication Paradigms Beteween Academic and Clinical Service Institutions in Nursing. Iranian Journal of Medical Education: 11(6); 554-568

Heshmati-Nabavi F, Vanaki Z. (2010). Professional approach: the key feature of effective clinical educator in Iran.

Islamic Republic of Iran Ministry of Health and Treatment and Medical Education Supreme Council for Planning of Medical Sciences, Educational programs of Baccalaureate Degree operating room, Profile, program, curriculum and evaluation method. Adopted by 35 Th session of the Higher Council for Planning of Medical Sciences: 20 /12/2007.

Kenton, K. (2006). How to Teach and Evaluate Learners in the Operating Room. Obstetric Gynecology Cling N Am; 33; 325332.
Khazaei, J, Zarouj, H.Abbasi A. (2014). Qualification of Clinical Education of operation room in Bachelor degree from the perspective of students and educators in this field at the University of Medical Sciences, in Semnan Journal of Education and Ethics in Nursing; Volume 3, Number 3, 39-46 [In Persian]

Khazaei, J, Zarouj, H.Abbasi A. (2014). Qualification of Clinical Education of operation room in Bachelor degree from the perspective of students and educators in this field at the University of Medical Sciences, in Semnan Journal of Education and Ethics in Nursing; Volume 3, Number 3, 39-46 [In Persian]

Laura, C; Skoczylas, E B; Littleton, Steven, L; Kanter and Gary Sutkin. (2012). Teaching Techniques in the Operating Room: The Importance of Perceptual Motor Teaching. Academic Medicine; 87(3).

Lingard, L; Reznick, R; Espin, S; More. (2002). Team Communications in the Operating Room: Talk Patterns, Sites of Tension, and Implications for Novices. Academic Medicine; 77(3):232237.

Manninen E (1998) Changes in nursing students' perceptions of nursing as they progress through their education. J Adv Nurs 27: 390-8

Mayring p. (2000). Qualitative Content Analysis. Forum: Qualitative Social research; 1(2): 25-30

Moussaoui, S. (2005). Professional problems from the perspective of employees working in operating rooms. Hamedan University of Medical Sciences Journal; 12(47)

0’Driscoll M, Allan H, Smith P. (2010). Still looking for leadership-Who is responsible for student nurses' learning in practice? Nurse Education Today; 30(3):212-7

Omidvar S, Bakouee F, Salmalian H. (2010). Clinical education problems: the viewpoints of midwifery students in Babol University of Medical Sciences. Iranian Journal of Medical Education; 5(2):15-. [Persian]

Operating room and anesthesia, Introduction of operation room. Available from: URL; http:// www.mums.ac.ir/ornanesn/ fa/historyOR. Accessed: 27/5/2017

Riley R, Manias E (2002) Foucault could have been an operating room nurse. J Adv Nurs 39: 316-24 -15

Sadeghi A (2011). "A case study: the association between organizational culture with management knowledge in Hasheminezhad hospital - Tehran. Official Journal of the organs of administration of the hospitals in Iran, Issue 2, summer. No.37:1-8

Tanner J, Timmons S (2000) backstage in the theatre. J Adv Nurs 32: 975-80

Tazakori, Z. Mehri, S.Mobaraki, N.Dadash L. Ahmadi Y. Shokri, F.Hamrang, L. (2016). Factors Affecting on Quality of Clinical Education from Perspectives of Operating Room Students. Journal of Health and Care; 17 (2): 128 -136. [In Persian]

Zeighami R Faseleh M Jahanmiri Sh Ghodsbin F. (2004). Nursing student's viewpoint about problems of clinical teaching. The Journal of Ghazvin University of Medical Science; 8(30): 51-5. [Persian] 